\title{
Impact of Geometry Faceting on Air Flows at the Surface of Building Facades
}

\author{
Ajith Rao \\ USG \\ Chicago, USA
}

\begin{abstract}
Understanding air flows near the surface of building facades can lead to designing buildings that can better manage ventilation flows, wind loads and wind energy capture. Streamlined geometries impact air flows differently than bluff geometries, and are preferable from an air flow perspective, however they carry penalties in terms of their construction. In this paper, computational fluid dynamics simulations are used in a series of simulation experiments to examine the air flow characteristics at the surface of the geometries. Flow around a smooth cylindrical profile is compared to that around profiles with increasingly large facet sizes. The air flow patterns and dynamic pressure profiles at the surface are used as a means of comparison of the different geometric types. The results demonstrate the minimum size of faceting that can be utilized to approximate a completely smooth geometry, for air flow purposes.
\end{abstract}

Keywords- Air Flows, Computational Fluid Dynamics, CFD, Buildings

\section{INTRODUCTION}

Building forms can be categorized into two broad types for purposes of how air flows behave when it encounters the building: "bluff" and "streamlined" (Figure 1). Flows around bluff buildings are often complex, with separation and stagnation zones, turbulent wakes and vortices [1]. In comparison, the streamlined geometries demonstrate lesser degrees of separation and vortex formation. Understanding the nature of these flows is critical as this can help with managing ventilation flows, wind loads on buildings, and wind energy capture [2].

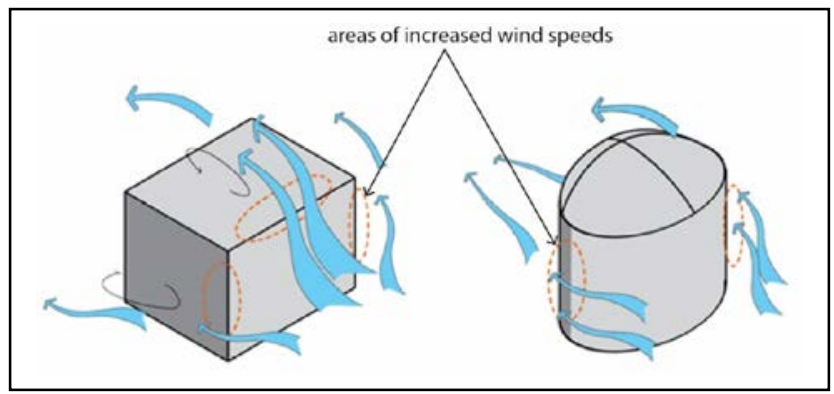

Fig. 1. Image showing "bluff” (left) and "aerodynamic” (right) building forms, with air flow patterns around them

Bluff building forms are generally easier to construct, and carry lower construction costs; while streamlined forms may be desirable from an air flow perspective, but create challenges in terms of construction systems required and associated costs, because of the use of single- or double-curved panels or forms. This raises the question as to whether the surfaces in a streamlined form need to be completely smooth or whether they can be faceted to some degree, to reduce the amount of separation caused from the sharp edges (Figure 2). This question has important ramifications for the construction techniques that need to be adopted for these geometries on the building scale, and consequently the cost of building the streamlined design.

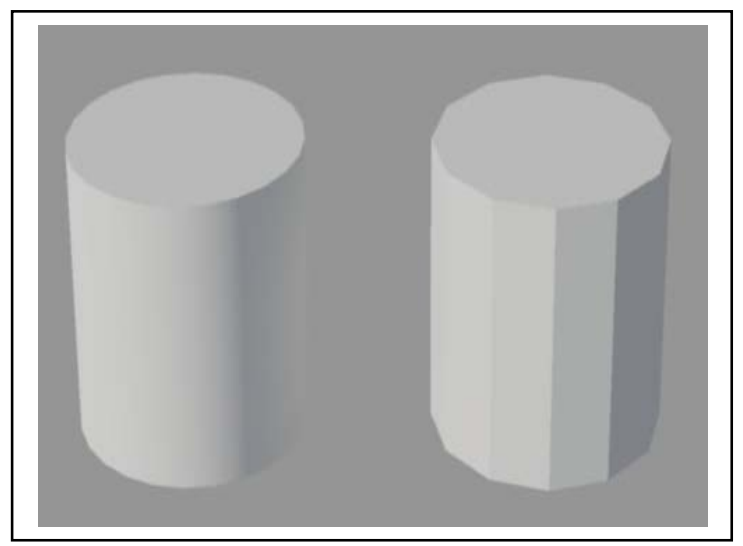

Fig. 2. This paper explores the question of what sized faceting (right) can reasonably approximate a completely smooth surface (left) with minimal impacts on air flow behavior

In this paper, Computational Fluid Dynamics (CFD) is employed in a series of simulation experiments to examine the flow characteristics at the surface of the geometries. Flow around a smooth cylindrical profile is compared to that around profiles with increasingly large facet sizes. The flow pressure profiles at the surface are used as a means of comparison of the different geometric types.

\section{EXPERIMENTAL SETUP}

Airflow around a completely smooth circular twodimensional geometry is compared to similar sized circular geometries faceted to various degrees. Air flow patterns and dynamic pressure near the surface is used as a means of comparison between the different geometries. If the amount of dynamic pressure does not vary significantly for the faceted geometries when compared to the smooth geometry, it means that the faceting has minimal or no impact on the airflow at that point.

This experiment involved a series of CFD simulations that examined the flow around five dimensional circular shapes that were faceted to various degrees of roughness, and compared to a completely smooth circular shape. Altogether, six different shapes were analyzed, as shown in Figure 3. The radius of all the shapes was maintained constant at $20 \mathrm{~m}$., for means of comparison. For each of the faceted options, the angles between the adjacent facets are shown in Figure 4. 


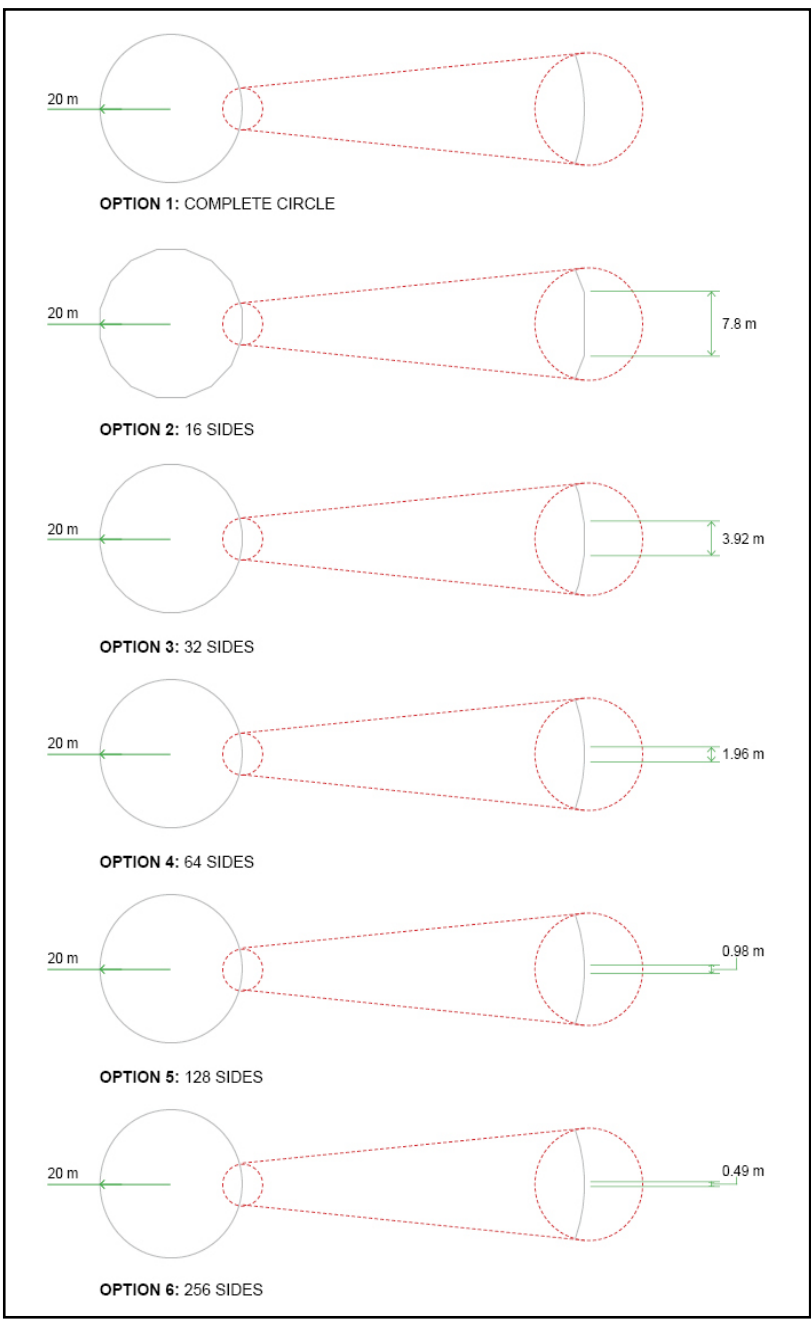

Fig. 3. Different sizes of the individual facets within the faceted geometries utilized for the experiment

The geometries were created using a CAD software, AutoCAD, and exported to a CFD software, Fluent [3], in the ACIS format. The overall dimensions and boundary conditions of the analysis domain used are in Figure 5.

A triangular mesh with the pave function was used, as shown in Figure 6. A boundary layer was provided on the edge of the shape, to closely monitor the flows in the area of interest. The grid size near the surface was kept at $0.1 \mathrm{~m}$.

For the CFD simulations, a double precision solver was used. The standard k- $\varepsilon$ turbulence model was adopted in all the cases. Enhanced wall treatment with pressure gradient effects was provided, to increase the accuracy of flows close to the surface. The second order upwind discretization scheme was used for momentum, $\mathrm{k} \& \varepsilon$. A constant input wind speed of 4 $\mathrm{m} / \mathrm{s}$ was used in all the cases. A convergence criterion of 0.001 was used for the residuals.

The results were analyzed for the air flow velocities and pressure close to the surface (within a maximum distance of 2$5 \mathrm{~m}$ ). If any of the faceted surfaces do not show any difference from the smooth surface in terms of these two variables, then it could be reasonably assumed that the faceting does not create a significant impact.

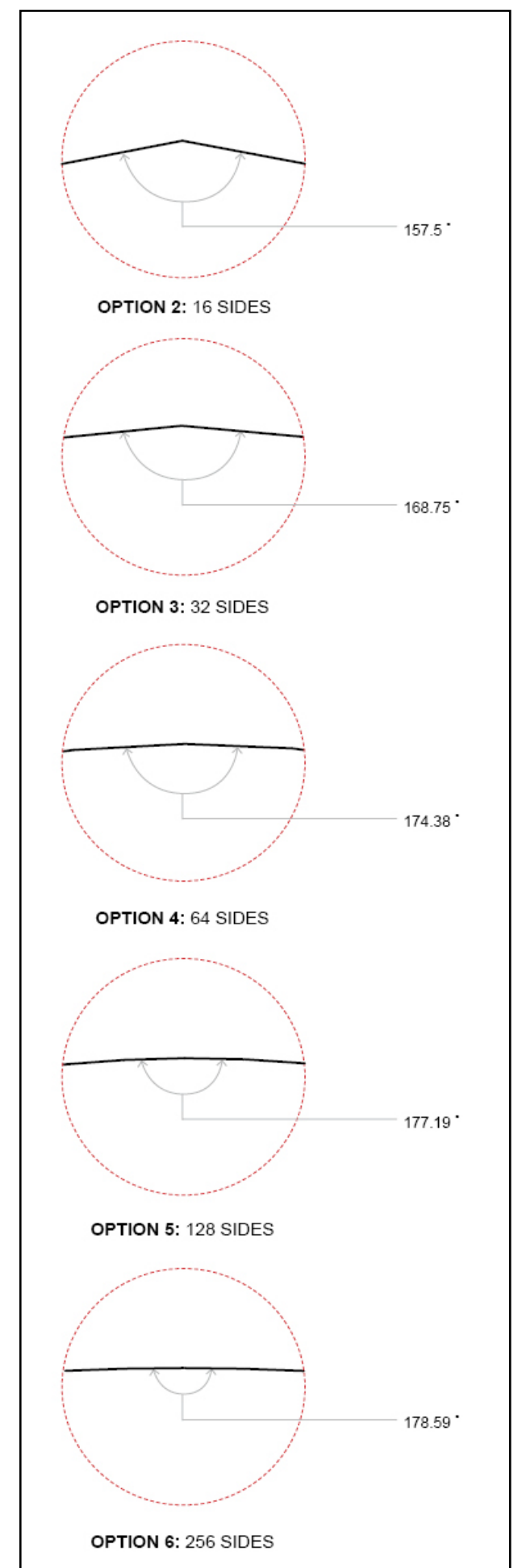

Fig. 4. Angle between the adjacent facets in the faceted geometries

A grid independence study is carried out to validate the results. The results are tested for three different grid size variations with a resolution difference of $15 \%$ between them, and compared. 


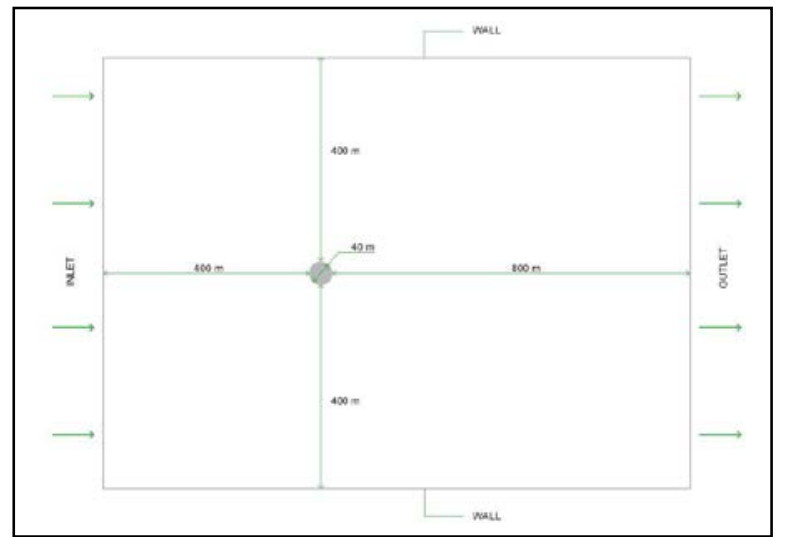

Fig. 5. Overall dimensions and boundary conditions used for the simulation models

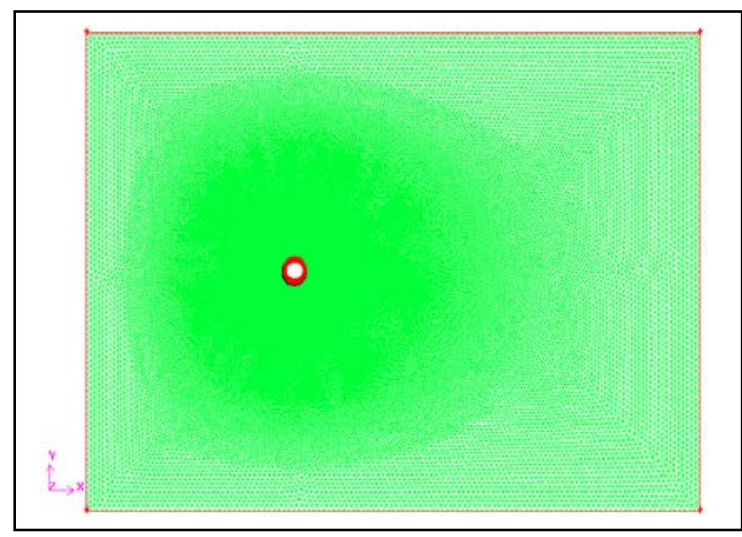

Fig. 6. Meshing grid utilized for the simulation models

\section{RESULTS}

In addition to examining the airflow patterns, dynamic pressure across the surface of the shapes was also plotted. The air flow and dynamic pressure for all the options are shown in Figures 7-13.

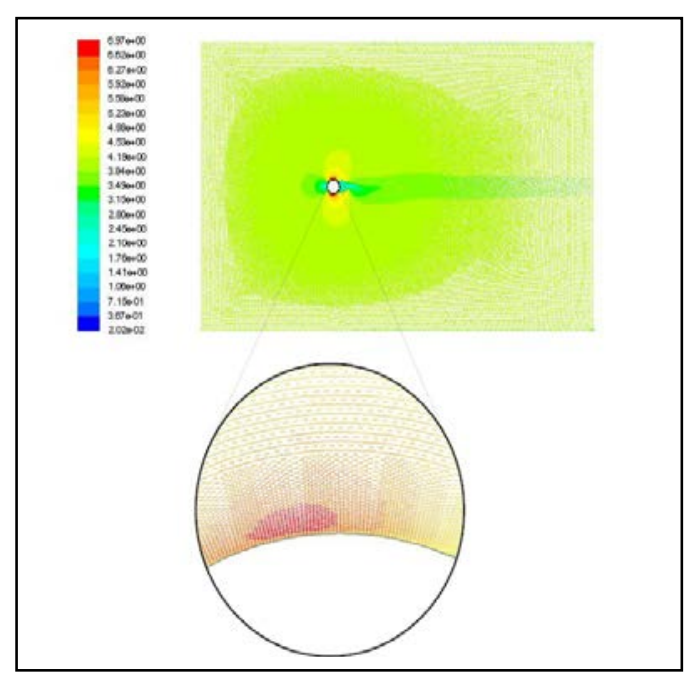

Fig. 7. Air flow patterns around Option 1 (completely smooth geometry)

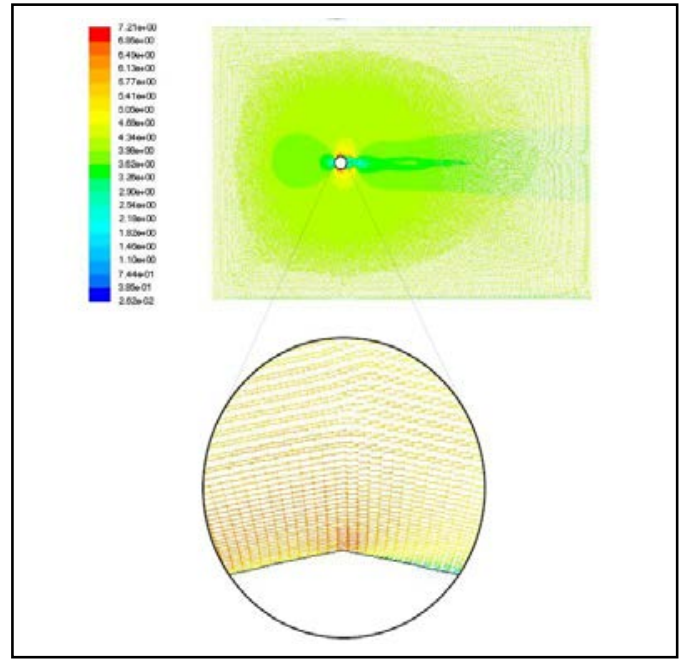

Fig. 8. Air flow patterns around Option 2 (Facet size $=7.8 \mathrm{~m}$ )

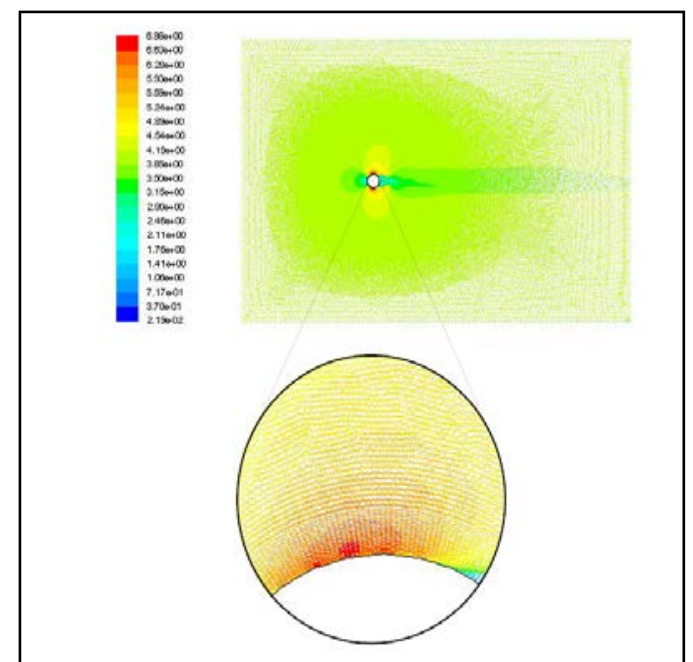

Fig. 9. Air flow patterns around Option 3 (Facet size $=3.92 \mathrm{~m}$ )

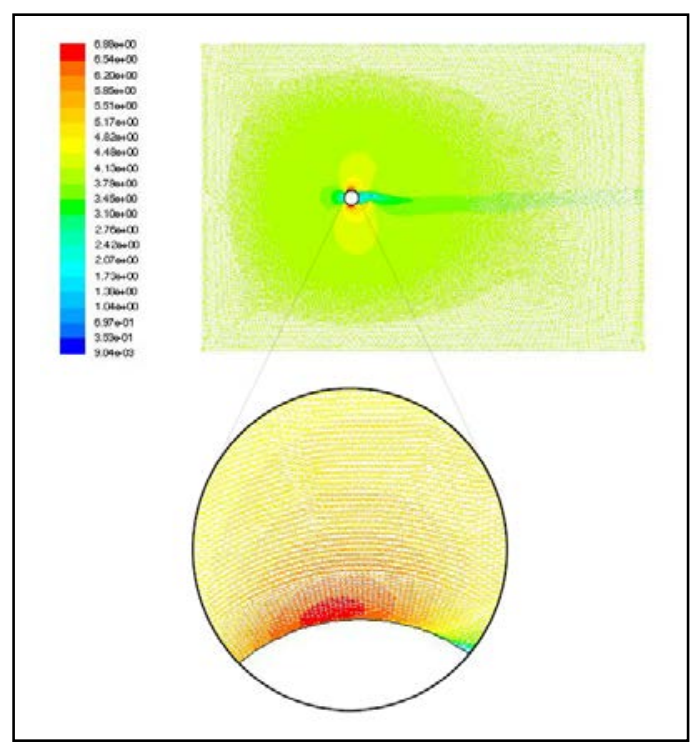

Fig. 10. Air flow patterns around Option 4 (Facet size $=1.96 \mathrm{~m}$ ) 


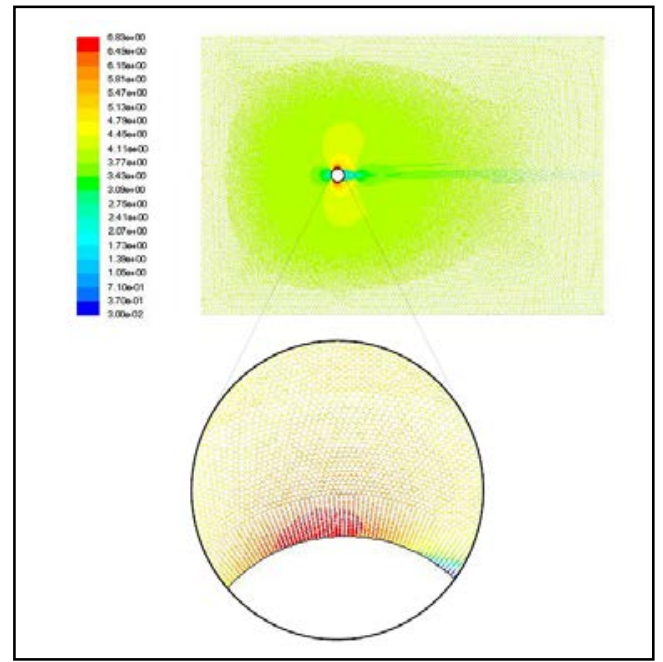

Fig. 11. Air flow patterns around Option 5 (Facet size $=0.98 \mathrm{~m}$ )

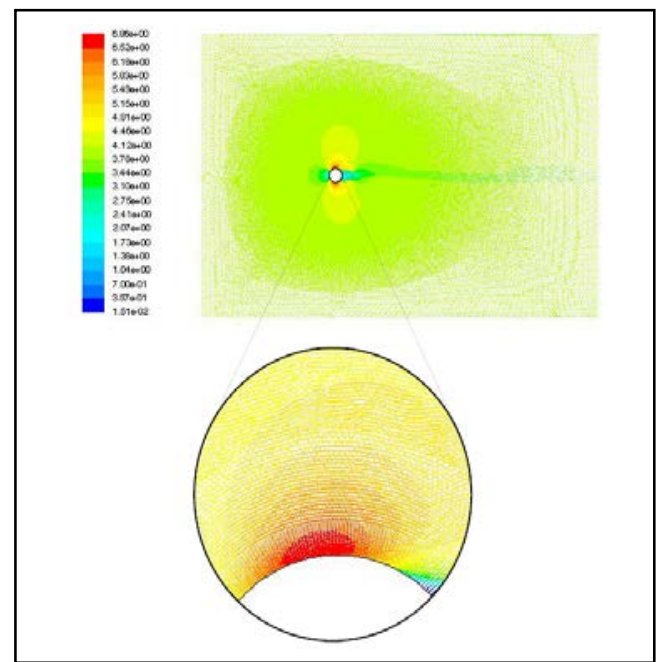

Fig. 12. Air flow patterns around Option 6 (Facet size $=0.49 \mathrm{~m}$ )

\section{CONCLUSIONS}

The experiments explored the effects of faceting for a circular geometry, with a radius of $20 \mathrm{~m}$. The results showed that at 128 divisions (i.e., a facet size of $0.98 \mathrm{~m}$ ), the effects of faceting are not consequential. This size can be rounded up to $1.0 \mathrm{~m}$ for similar results. At 256 divisions (i.e. a facet size of $0.49 \mathrm{~m}$ ), the surface behaves almost exactly like its circular counterpart. In conclusion, it is shown that a completely smooth geometry can be faceted without noticeable impacts on air flows near the surface.

Converting these lengths into a percentage value of the circumference, it shows that if the facet size needs to be at least equal to or lesser than approximately $0.79 \%$ of the length of the circumference. It must be noted that the length of the facet needs to be considered in conjunction with the angle between two adjacent facets, especially when the geometry is completely circular.

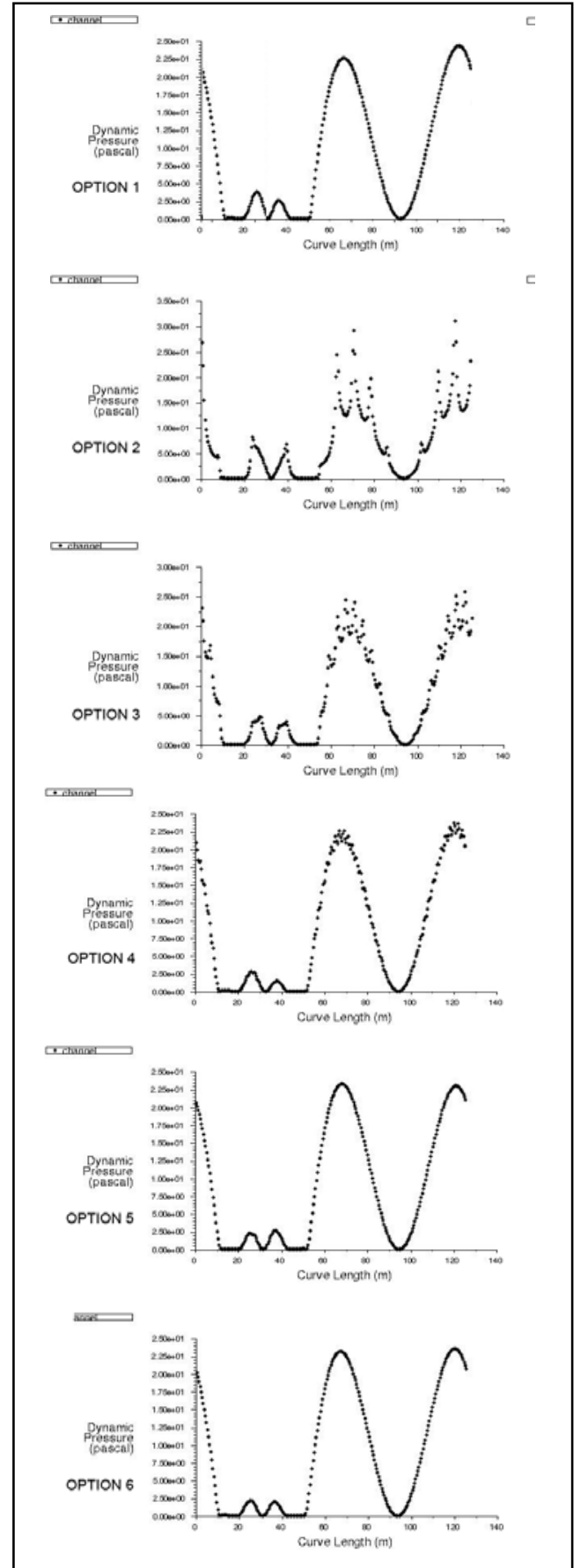

Fig. 13. A comparison of dynamic pressure along the shapes shows that the 128- and 256-sided shapes (Options 5 \& 6) are virtually similar to the circular shape (Option 1)

\section{REFERENCES}

[1] Cermak, J.E., "Aerodynamics of Buildings”, in Annual Review of Fluid Mechanics, Volume 8, 1976, pp. 75-106.

[2] Stathopoulos, T., "Wind Effects on Structures: Fundamentals and Applications to Design,” 3rd Edition, Wiley, New York, 2007.

[3] FLUENT Incorporated, Fluent User's Guide, Lebanon NH, 1998. 